adherence=1) and EDS impact satisfaction with healthcare providers (HCPs) and overall OSA care. Due to no adjustments for multiplicity, P-values are nominal.

Results Of 2289 participants $(50.3 \%$ female; mean \pm SD 44.8 \pm 11.1 years old; $42.5 \%$ ESS $>10$ ), $72 \%$ were satisfied with HCPs and $65 \%$ with OSA care. In the nonuse $(n=700)$, nonadherent $(n=153)$, intermediate $(n=225)$, and highly adherent $(\mathrm{n}=1211)$ groups, 47\%, 52\%, 53\%, and $36 \%$ had EDS, respectively. An additional h/night PAP use was associated with lower ESS scores $(n=1589, \beta=-0.28 ; 95 \%$ confidence interval $[C I]=-0.40,-0.16$; $P<0.001)$. In PAP users $(n=1589)$, adherence associated with higher satisfaction with HCPs (adjusted odds ratio $[\operatorname{adjOR}]=2.37 ; 95 \% \mathrm{CI}=1.64,3.43 ; \mathrm{P}<0.001)$ and OSA care $(\operatorname{adjOR}=2.91 ; 95 \% \mathrm{CI}=2.03,4.17 ; \mathrm{P}<0.001)$, and EDS with less satisfaction with $\mathrm{HCPs}$ (adjOR $=0.62 ; 95 \%$ $\mathrm{CI}=0.48,0.80 ; \mathrm{P}<0.001)$ and OSA care $(\operatorname{adjOR}=0.50 ; 95 \%$ $\mathrm{CI}=0.39,0.64 ; \mathrm{P}<0.001)$.

Discussion In this real-world study, EDS was prevalent among highly adherent PAP users. PAP adherence was associated with greater patient satisfaction with HCPs and overall care, whereas persistent EDS was associated with lower patient satisfaction with HCPs and overall OSA care.

\section{HOW CAN HEALTH COACHING IMPROVE COMPLIANCE IN CPAP PATIENTS AND DOES IT WORK?}

Punam Korat*. Cardiff and Vale NHS Health Board, Cardiff, UK

\subsection{6/bmjresp-2021-bssconf.14}

Introduction Health coaching/patient initiation is part of the NHS long term plan. This case study will look at the way health coaching can help in improving compliance and way we can implement it in our service.

Case Study 62yr old female who was set up on CPAP due to severe OSA. Had been compliant at the beginning from Average hours of $6.5 \mathrm{hrs}$, AHI $2.3 / \mathrm{hr}$ when using CPAP and a mask leak of $0.5 \%$, but then compliance had dropped. Patient seemed to have been struggling with mask fit and therefore was issued with several different masks.

Came into a compliance clinic and still using CPAP sporadically and no improvement in compliance. Coaching conversation started and patient then began explaining the main reasons which were stopping her from using her CPAP. Goals were set for the patient to achieve before her next appointment.

Method Initiating the coaching conversation did improve the patients compliance and she began feeling the benefits of her treatment and felt more positive towards it.

Conclusion Need to start having conversations and asking key questions to consistent non-compliant patients which may uncover the root cause and engaging the patient to take control of their therapy and getting patients to find their own answers.

\section{A SYSTEMATIC REVIEW OF THE TREATMENT OF RESTLESS LEG SYNDROME AND PERIODIC LIMB MOVEMENTS IN PEOPLE WITH SPINAL CORD INJURY}

1,2Susan (sue) Cross*, ${ }^{3}$ Nicola Barclay. ${ }^{1}$ National Spinal Injuries Centre, Stoke Mandeville, Bucks. England., Aylesbury, UK; ${ }^{2}$ MSc Sleep Medicine graduate, Sleep and Circadian Neuroscience Institute, Dept. of Clinical Neurosciences, University of Oxford, UK; ${ }^{3}$ Supervising tutor, Sleep and Circadian Neuroscience Institute, Dept. of Clinical Neurosciences, University of Oxford, UK

\subsection{6/bmjresp-2021-bssconf.15}

Introduction People with spinal cord injury (SCI) have a high prevalence of sleep disorders, which often remain undiagnosed and untreated, resulting in reduced quality of life. Their sleep may be disturbed by pain and spasms, but these could also be symptoms of Restless Leg Syndrome (RLS) or Periodic Limb Movements (PLMs). Studies estimate that RLS affects 17-19\% of people with SCI. Little is known about the management of RLS or PLMs after SCI.

Method A systematic review was conducted following PRISMA guidelines, searching 5 databases, for studies of any intervention to manage RLS and/or PLMs in people with SCI. Outcomes of interest were changes in severity of RLS or PLMs, or effects on sleep quality (see table 1).

Results 465 Manuscripts were identified. 12 studies fulfilled inclusion/exclusion criteria, totalling 108 participants. Risk of bias assessment revealed low evidence quality due to small sample sizes and lack of randomised controlled trials.

Interventions investigated included - L-dopa and pramipexole were shown to reduce PLMs objectively measured by polysomnography, and subjectively reported RLS symptoms.

- Exercise reduced the PLM index measured by polysomnography.

- Intrathecal baclofen injection reduced PLMs.

Discussion It is thought that in people with SCI dopaminergic medications exert their effect in the spinal cord rather than in the brain.

Reviewed papers report potential confusion between PLMs and spasticity, and between neuropathic pain and RLS.

The RLS diagnostic criteria are challenging to apply after SCI. It is unclear how a patient with altered sensation following SCI will experience the 'urge' to move.

Conclusions There is evidence that some sleep measures improved with dopaminergic medication or exercise. Clinicians should consider investigation for RLS and PLMs in patients with neuropathic pain or spasticity unresponsive to usual treatment, with symptoms predominating in the evening or at night. These patients may respond to treatment for these sleep disorders.

Abstract 17 Table 1 Terms used in healthcare databases advanced search' (HDAS) function in OpenAthens

\begin{tabular}{|c|}
\hline spinal cord injur* or spinal injur* or SCl or tetraplegia or quadriplegia or paraplegia \\
\hline AND \\
\hline periodic limb movement* or PLM or PLMS or PLMD or restless leg or RLS or Willis-Ekbom Disease \\
\hline
\end{tabular}

